\title{
Analysis of the genetics of boar taint reveals both single SNPs and regional effects
}

\author{
Suzanne J Rowe ${ }^{1,7 *}$, Burak Karacaören ${ }^{1,2}$, Dirk-Jan de Koning ${ }^{1,3}$, Boris Lukic ${ }^{4}$, Nicola Hastings-Clark', Ingela Velander ${ }^{5}$, \\ Chris S Haley ${ }^{1,6}$ and Alan L Archibald ${ }^{1}$
}

\begin{abstract}
Background: Boar taint is an offensive urine or faecal-like odour, affecting the smell and taste of cooked pork from some mature non-castrated male pigs. Androstenone and skatole in fat are the molecules responsible. In most pig production systems, males, which are not required for breeding, are castrated shortly after birth to reduce the risk of boar taint. There is evidence for genetic variation in the predisposition to boar taint.

A genome-wide association study (GWAS) was performed to identify loci with effects on boar taint. Five hundred Danish Landrace boars with high levels of skatole in fat $(>0.3 \mu \mathrm{g} / \mathrm{g})$, were each matched with a litter mate with low levels of skatole and measured for androstenone. DNA from these 1,000 non-castrated boars was genotyped using the Illumina PorcineSNP60 Beadchip. After quality control, tests for SNPs associated with boar taint were performed on 938 phenotyped individuals and 44,648 SNPs. Empirical significance thresholds were set by permutation $(100,000)$. For androstenone, a 'regional heritability approach' combining information from multiple SNPs was used to estimate the genetic variation attributable to individual autosomes.
\end{abstract}

Results: A highly significant association was found between variation in skatole levels and SNPs within the CYP2E1 gene on chromosome 14 (SSC14), which encodes an enzyme involved in degradation of skatole. Nominal significance was found for effects on skatole associated with 4 other SNPs including a region of SSC6 reported previously. Genome-wide significance was found for an association between SNPs on SSC5 and androstenone levels and nominal significance for associations with SNPs on SSC13 and SSC17. The regional analyses confirmed large effects on SSC5 for androstenone and suggest that SSC5 explains 23\% of the genetic variation in androstenone. The autosomal heritability analyses also suggest that there is a large effect associated with androstenone on SSC2, not detected using GWAS.

Conclusions: Significant SNP associations were found for skatole on SSC14 and for androstenone on SSC5 in Landrace pigs. The study agrees with evidence that the CYP2E1 gene has effects on skatole breakdown in the liver. Autosomal heritability estimates can uncover clusters of smaller genetic effects that individually do not exceed the threshold for GWAS significance.

Keywords: Boar taint, Skatole, Androstenone, Regional heritability, Genome-wide association

\section{Background}

Boar taint is an offensive urine or faecal-like odour, affecting the smell and taste of some cooked pork. Androstenone and skatole, which are lipophilic compounds that accumulate in the fat of mature non-castrated male pigs,

\footnotetext{
* Correspondence: suzanne.rowe@agresearch.co.nz

'The Roslin Institute and R(D)SVS, University of Edinburgh, Easter Bush, Midlothian, Scotland EH25 9RG, UK

${ }^{7}$ AgResearch, Dept of Animal Genomics, Invermay Agricultural Centre, Private Bag 50034, Puddle Alley, Mosgiel 9053, New Zealand

Full list of author information is available at the end of the article
}

have been identified as the main causes of boar taint [1]. A range of thresholds, above which negative reactions from consumers are expected, have been reported for androstenone $(>0.5-1.0 \mu \mathrm{g} / \mathrm{g}$ fat $)$ and skatole $(>0.2-0.25 \mu \mathrm{g} / \mathrm{g}$ fat $)$ [2-6]. The scale of the problem was revealed in a large EU study of carcasses from over 40,000 non-castrated male pigs. Androstenone levels exceeded $1.0 \mu \mathrm{g} / \mathrm{g}$ fat and skatole levels exceeded $0.25 \mu \mathrm{g} / \mathrm{g}$ fat in $30 \%$ and $11 \%$ of these carcasses, respectively [3]. The cost of testing, losses in carcass value and potential future lost sales result in a substantial economic cost to the industry.

\section{Biomed Central}

(c) 2014 Rowe et al.; licensee BioMed Central Ltd. This is an Open Access article distributed under the terms of the Creative Commons Attribution License (http://creativecommons.org/licenses/by/2.0), which permits unrestricted use, distribution, and reproduction in any medium, provided the original work is properly credited. 
Androstenone or $5 \alpha$-androst-16-en-one is a male steroid produced in the testes at sexual maturity. High concentrations of androstenone are present in the saliva of male pigs where it is converted to a pheromone and is an important olfactory trigger for sexual behaviour in sows [7]. Androstenone accumulates in adipose tissue producing taint when the fat is heated. The ability to detect this taint is itself under genetic control in humans and largely governed by the OR7D4 receptor. Approximately $70 \%$ of the human population are unable to detect the associated urine like odour [8,9]. Skatole or 3-methyl-indole is produced from the breakdown of tryptophan by bacteria in the hindgut of the pig and subsequently absorbed into the blood stream where it is largely metabolised in the liver and excreted in urine. Skatole which is not degraded in the liver is deposited in peripheral tissues mainly accumulating in adipose tissue.

The most effective solution, to date, for controlling boar taint, is surgical castration shortly after birth. However, as castration removes natural anabolic androgens that promote lean growth, non-castrates are leaner with $10-30 \%$ greater efficiency in feed conversion and superior meat quality. Furthermore, concerns over animal welfare have led to legislative control [10]. Within Europe an industry-wide agreement is in place to cease castration for welfare reasons by 2018 (http://ec.europa.eu/food/animal/ welfare/farm/initiatives_en.htm), forcing the industry to explore other methods to prevent tainted carcasses.

Selective breeding based on the identification and exploitation of genetic variation in androstenone and skatole levels could ultimately provide a more sustainable solution [11]. Recent studies have revealed Quantitative Trait Loci (QTL) with effects on skatole or androstenone, including QTL mapped to almost every chromosome [11-18]. The genetic architecture of predisposition to boar taint shows evidence for inter- and intra-breed variation with many of the reported effects appearing to be breed specific [11,16-19]. In general, Duroc pigs tend to have high levels of androstenone, and the Landrace breeds high levels of skatole. The relationship between the two compounds is complex. Testicular steroids have been shown to inhibit the breakdown of skatole in the liver but the relationship between the compounds and the underlying mechanisms are not well understood [20].

Although highly successful at identifying new trait associated loci and pathways, human genome-wide association studies (GWAS) have failed to capture a large proportion of the genetic variation in complex traits $[21,22]$. To address this so-called 'missing heritability' gap, methods have been developed involving the analysis of larger regions of the genome to account for variation unexplained by analysis of individual single nucleotide polymorphisms (SNPs) [23]. Estimating local heritability using larger regions captures additive variation in the genome which might elude the stringent significance thresholds necessary for testing each SNP individually. It has also been suggested that rare variants not in complete linkage disequilibrium (LD) with common SNP markers are captured by estimating the genetic variation from an entire "region" or set of SNPs [24].

The objective of this study was to identify genomic regions with effects on boar taint in Landrace pigs.

Results are reported from the two approaches used: (i) single SNP analysis using genome-wide association, and (ii) a regional approach dividing SNPs by chromosome and estimating genetic variation attributable to each autosome.

\section{Results}

We acquired data for a population of approximately 6,000 commercial Danish Landrace boars. The animals were slaughtered at a mean age of $160( \pm 13)$ days. Measures for skatole were taken using an in-line procedure at three Danish abattoirs. Power to detect a QTL can be increased in a finite sample by selecting those individuals that differ most from the phenotypic mean i.e. the extremes of the phenotypic distribution. Here, we took extreme animals plus a within-litter 'control' in order to maximize power while controlling for family stratification. This strategy maximises the potential genetic information to be gained from the sample $[25,26]$. Thus, 500 boars with high skatole $(>0.3 \mu \mathrm{g} / \mathrm{g}$ fat) at slaughter, each matched with a low skatole litter mate (the lowest in the litter and in any event below $0.3 \mu \mathrm{g} / \mathrm{g}$ ) were selected for genome-wide analysis. Phenotypic measurements for androstenone in adipose tissue were subsequently collected for these selected 1,000 boars.

The measures for both skatole and androstenone were positively skewed and were log transformed prior to analysis (Additional file 1: Figure S1). Descriptive statistics and heritabilities for both traits are given in Table 1. Pedigree information and skatole measures were available for 5,000 boars from the initial population that were not selected for genotyping and genome-wide analyses. Narrow sense heritabilities estimated from pedigree rela-

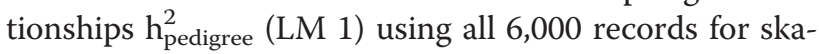
tole and 1,000 records for androstenone were moderate at 0.39 (s.e. 0.03 ) and 0.52 (s.e. 0.09) respectively and were similar to those previously reported [16,27]. The genomic heritability estimate of 0.07 (s.e. 0.01) for skatole in the selected individuals was very low (Table 1 ). This result was expected and reflects the experimental design as the selected individuals comprised phenotypically divergent sibs for skatole thus maximising the within family variance. Narrow sense heritability is based on a ratio of the between and within family variance and is therefore reduced (and was similarly reduced in the pedigree based estimate using only the 1,000 genotyped 
Table 1 Descriptive statistics for skatole and androstenone

\begin{tabular}{|c|c|c|}
\hline & Skatole & Androstenone \\
\hline Mean $(\mu \mathrm{g} / \mathrm{g})$ & 0.32 & 1.05 \\
\hline $\mathrm{Sd}(\mu \mathrm{g} / \mathrm{g})$ & 0.24 & 0.93 \\
\hline Range $(\mu \mathrm{g} / \mathrm{g})$ & $0.02-2.49$ & $0.06-9.23$ \\
\hline $\begin{array}{l}\text { Effect of slaughter weight } \\
\text { (sig of effect) })^{T}\end{array}$ & $-0.0089(3.43 \mathrm{E}-05)$ & $-0.023(0.0007)$ \\
\hline $\begin{array}{l}\text { Effect of meat percentage } \\
\text { (sig of effect })^{\dagger}\end{array}$ & $-0.048(9.1 \mathrm{E}-12)$ & $-0.026(0.06)$ \\
\hline$h_{\text {pedigree }}^{2}(\mathrm{se})$ & $0.39(0.03)^{t+}$ & $0.52(0.09)$ \\
\hline$h^{2}$ snp $(s e)$ & $0.07(0.01)$ & $0.35(0.08)$ \\
\hline
\end{tabular}

Data from 938 progeny of 128 sires and 441 dams.

${ }^{\dagger}$ Covariate effects estimated in LMM using log trait.

$\mathrm{h}_{\text {pedigree }}^{2}$ refers to narrow sense heritability estimated in a linear mixed model using GRM estimated from pedigree relationships.

${ }^{+\top}$ Narrow sense heritability estimated for skatole using pedigree relationships from 6000 individuals.

$\mathrm{h}_{\mathrm{SNP}}^{2}$ refers to narrow sense heritability estimated in a linear mixed mode using GRM estimated from SNP genotypes.

individuals (not shown)). Comparing variance components estimated from the unselected and selected populations provides an indication of how effects estimated in the selected sample would scale to the population as a whole. Mean skatole measures for selected boars and their litter mates were 0.48 (sd. 0.25) and 0.15 (sd. 0.06) $\mu \mathrm{g} / \mathrm{g}$ respectively. Although data were selected for skatole, androstenone measures also differed slightly (but not significantly) between the two groups with a mean of 1.25 (sd. 1.0) $\mu \mathrm{g} / \mathrm{g}$ in the high skatole animals, and 0.85 (sd. 0.77) $\mu \mathrm{g} / \mathrm{g}$ in their low skatole litter mates. The estimated genetic correlation between skatole and androstenone in the selected data was 0.27 (s.e. 0.20). Because the estimate of the additive genetic variance in skatole is biased downwards in the genotyped subset, the genetic correlation between skatole and androstenone is also likely to be underestimated.

\section{Genome-wide association study (GWAS)}

DNA isolated from muscle samples collected at slaughter were genotyped for 63,153 SNPs using the Illumina PorcineSNP60 beadchip [28]. Analysis was restricted to the autosomes. The genotype data were subjected to quality control (QC) through an iterative process performed using the GenABEL package in R 2.9.1 software [29,30]. The QC criteria for SNPs were call rates $>0.95$ and minor allele frequencies $(\mathrm{MAF})>0.01$. The $\mathrm{QC}$ criteria for individuals were call rates $>0.95$, heterozygosity $<0.45$ ( $1 \%$ false discovery rate $(\mathrm{FDR})$ ) and identity-by-state (IBS) < 0.95 . After QC, 44,648 autosomal SNPs and 938 individuals were included in the final analysis. SNP locations throughout the analysis are given according to the published draft pig genome sequence (Sscrofa10.2: ftp://ftp.ncbi.nlm.nih.gov/genbank/genomes/ Eukaryotes/vertebrates_mammals/Sus_scrofa/Sscrofa10.2/)
[31] and as available in Ensembl release 75 (http://www. ensembl.org/Sus_scrofa/Info/Index).

\section{Population stratification}

Genome wide association is based on differences in allele frequencies associated with differences in the trait under study. Phenomena such as admixture, selection and population stratification can result in spurious patterns of allele frequencies unrelated to the trait. Population stratification can be assessed by clustering individuals based on measures of relatedness and examining clusters for evidence of systematic bias. Here, model based clustering was performed using the mclust function in R software 2.10.1 [30]. Mclust uses Bayesian information criterion (BIC) and an expectation maximisation algorithm (EM) to select the optimal model and number of clusters. The best fit for the data was 3 elipsoidal clusters (Figure 1). Multi-dimensional scaling (mds) was applied to a distance matrix obtained as

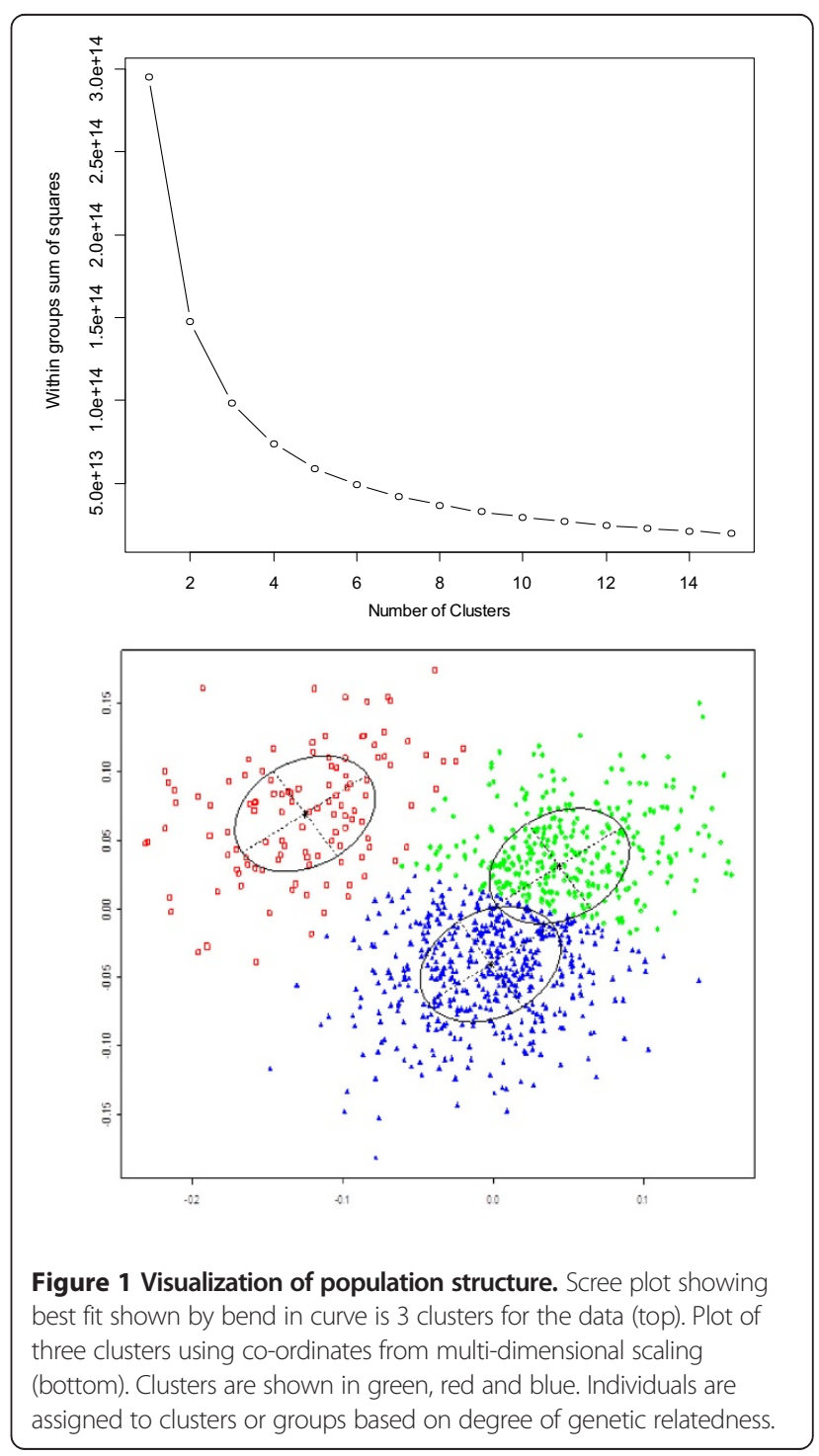


a function of the weighted genomic relationship matrix. Multi-dimensional scaling returns a matrix with $k$ columns whose rows give the coordinates of the points chosen to represent dissimilarities. $k$ is a user defined parameter based on the expected number of clusters, here $k=3$. The 3 columns from the mds matrix were fitted into the linear model as covariates in order to account for the population stratification indicated by the model based clustering.

The differences in study design between the two traits (i.e. skatole, androstenone) were reflected in the GWAS by very different estimates of lambda, which is an indicator of bias due to population structure. Lambda was close to 1 for all of the skatole analyses where high and low animals were matched sibs, but greater than 2 for the androstenone analyses. This result indicates that the sampling design for skatole was balanced and therefore, unaffected by potential biases arising from any population stratification. Although lambda indicates some bias for the androstenone analyses, this bias was largely accounted for with the inclusion of the co-ordinates from the multi-dimensional scaling (mds) matrix in the model (i.e. the inclusion of the mds matrix lowered the value of lambda from 2.0 to 1.3). Any remaining stratification was successfully corrected for by fitting the genomic relationship matrix. Full details are given in the materials and methods.

Single SNP associations were performed using a GRAMMAR [29] analysis (LM 3) in GenABEL software. The results are summarized in Figure 2. Test statistics exceeding genome-wide significance were found on SSC14 for skatole, and on SSC5 for androstenone. Further peaks on SSC13 and SSC17 exceed a genome-wide 5\% FDR for effects on androstenone. Effects on skatole exceeding nominal significance but not genome-wide significance were also seen on SSC3, SSC5, SSC6 and SSC8 (Table 2).

\section{Skatole}

The effect of the SIRIO000194 SNP at the telomeric end of SSC14 on skatole levels was highly significant $(P<1.4 \mathrm{E}-09)$ exceeding the genome-wide threshold (Figure 2) and explaining $\sim 5 \%$ of the phenotypic variance. This SNP lies within the CYP2E1 gene, which encodes an enzyme involved in the breakdown of skatole [32-34]. The next ranking SNP after the SNPs in LD with SIRI0000194 is the ASGA0039716 SNP on chromosome 8. The ASGA0039716 SNP lies within the gene TET2 or methylcytosine dioxygenase 2. There is no obvious connection between the function of this gene or any other protein coding genes within $1 \mathrm{Mbp}$ of TET2 as currently annotated in the pig genome and skatole metabolism or storage. SNPs on chromosomes 3,5 and 6 also reach nominal significance. When we fitted SIRI0000194 as a fixed effect the ranking changed and $M A R C 0040638$ was the top ranking SNP $(P<0.001)$.

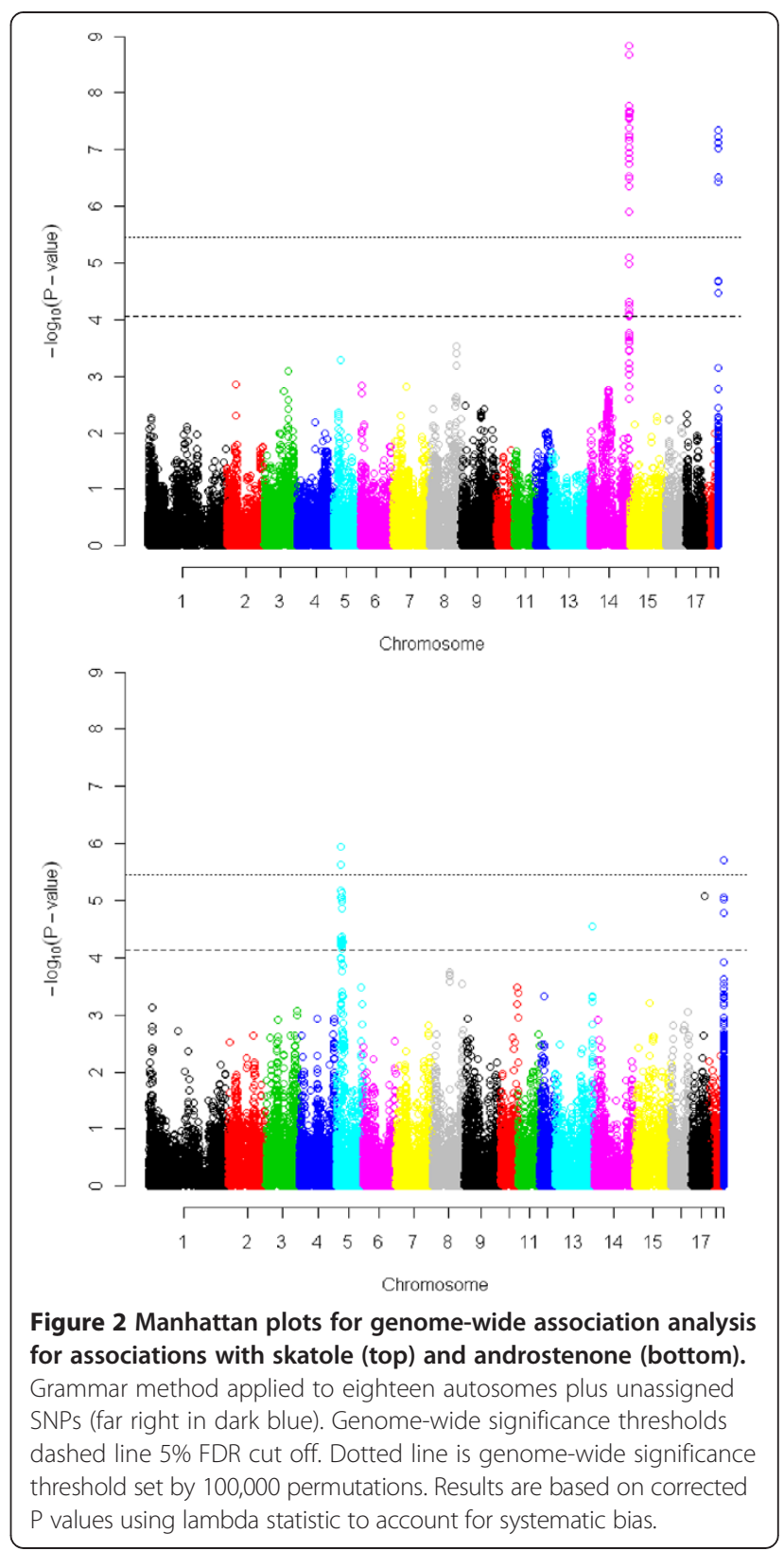

\section{Androstenone}

A peak of genome-wide significant SNP effects on androstenone was seen on SSC5 $(\mathrm{P}<6.8 \mathrm{E}-07)$ explaining $4 \%$ of the phenotypic variation (Table 2). Two SNPs H3GA0016037 and ASGA0025097 mapping $4 \mathrm{Mbp}$ apart are highly significant. Figure 3 shows the LD structure and genes around the SSC5 peak SNP for androstenone. LD between the two SNPs is relatively high at $r^{2}=0.68$ suggesting that both SNPs are tagging the same causal variant. There were also SNPs with large effects on chromosomes 8, 13 and 17 (Table 2). SSC13 and 17 exceeded the genome-wide false discovery rate. ALGA0073594 on SSC13 does not map to any known gene. ASGA0095898 on SSC17 lies within PTPRT or protein tyrosine phosphatase, 
Table 2 Descriptive statistics for most significant SNP effects

\begin{tabular}{|c|c|c|c|c|c|c|}
\hline Chr & SNP & Pos (bp)§ & $P$ value & SNP effect & Proportion phenotypic variance & Sig-full ${ }^{\dagger}$ \\
\hline \multicolumn{7}{|c|}{ Skatole } \\
\hline 14 & SIRI0000194 & $153,477,507$ & $1.40 \mathrm{E}-09^{* *}$ & -0.26 & 0.05 & 1.66E-10 \\
\hline 8 & ASGA0039716 & $125,083,628$ & 0.00029 & 0.04 & 0.001 & 0.0018 \\
\hline 5 & ASGA0025182 & $28,884,161$ & 0.00052 & 0.12 & 0.02 & 0.00011 \\
\hline 3 & ALGA0020313 & $103,881,028$ & 0.00082 & 0.17 & 0.01 & 0.0006 \\
\hline 6 & MARC0040638 & $4,515,061$ & 0.00144 & -0.13 & 0.01 & 0.00031 \\
\hline \multicolumn{7}{|c|}{ Androstenone } \\
\hline 5 & H3GA0016037 & $20,902,965$ & $6.82 \mathrm{E}-07^{* *}$ & 0.26 & 0.04 & 5.17E-07 \\
\hline 5 & ASGA0025097 & $24,354,867$ & $3.51 \mathrm{E}-06^{*}$ & 0.28 & 0.03 & 2.03E-06 \\
\hline 17 & ASGA0095898 & $50,429,537$ & $1.08 \mathrm{E}-05^{*}$ & -0.52 & 0.02 & 0.0001 \\
\hline 13 & ALGA0073594 & $203,892,414$ & $2.38 \mathrm{E}-05^{*}$ & -0.17 & 0.02 & 3.63E-05 \\
\hline 8 & ASGA0093454 & $80,694,489$ & 0.0002 & -0.22 & 0.02 & 0.00024 \\
\hline
\end{tabular}

*exceeds 5\% genome-wide false discovery rate ${ }^{* *}$ exceeds genome-wide significance threshold estimated from 100,000 permutations ${ }^{\dagger}$ significance when tested in linear mixed model using ASReml software. § SNP position in base pairs in the Sscrofa10.2 genome assembly.

receptor type T and ASGA0093454 on SSC8 lies within the FH2 domain containing 1 gene.

\section{Autosomal heritability}

The linear mixed model (2) can be extended to divide phenotypic variance into estimates of the genetic and environmental variance containing information from genotypes of a group of $N$ SNPs spanning a region. This method has been implemented in the GCTA software package and it has been shown that the method can be used to estimate genetic variation for any region of the genome [35]. We divided the pig genome into the 18 autosomes and jointly estimated the contribution to heritability of androstenone (Figure 4, Additional file 2: Table S1) from each autosome (6). The total heritability summed over all autosomes was 0.29 for androstenone. As with the total heritability, the autosomal heritabilities for skatole will be specific to the genotyped subset and underestimated for the unselected population due to the study design. For this reason we have omitted the results on skatole from the main text, but these results can be found in Additional file 3.

Individual LRT (likelihood ratio tests) for each chromosome for androstenone are detailed in Table 3. These were derived by the LRTpoly test comparing a linear mixed model fitting systematic or fixed effects and a GRM based on information from all SNPs with a model incorporating an additional variance component for the genetic variance attributable to all SNPs on a chromosome. This provides a test of whether inclusion of individual autosomes provides a better model of the variance than the overall relationship matrix (as might be the case if the individual chromosomes harbor a gene or genes of large effect on the trait). Estimates of the autosomal heritabilities for effects on androstenone for LRTpoly are summarised in Table 3.

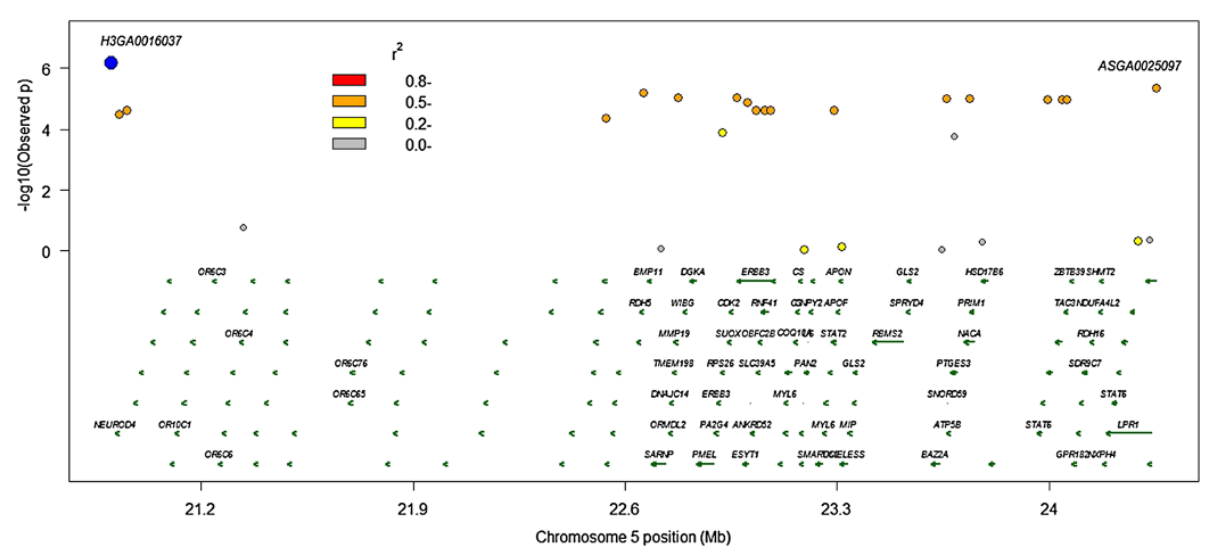

Figure 3 LD decay from SNP H3G000016037 plotted against significance of effect on androstenone, pairwise LD in the region and genes located within the region. Sscrofa genome build 10.2 . 


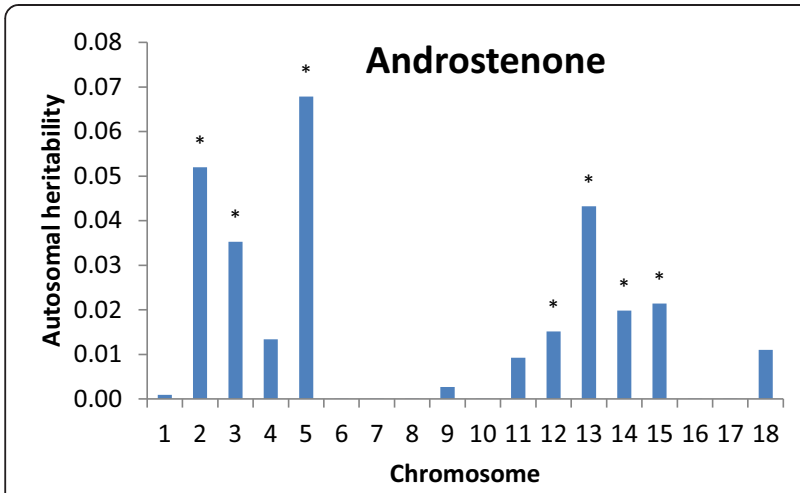

Figure 4 Autosomal heritability or proportion of phenotypic variance explained for androstenone. * estimate of heritability is larger than standard error. All 18 autosomes were fitted simultaneously in a mixed linear model.

For androstenone, the only autosome with a significant LRTpoly test for genetic variance was chromosome 5 explaining $6 \%$ of the phenotypic variation, reflecting the GWAS results. Under the LRTpoly method, autosomes 2, 3 and 13 each explain $2 \%$ of the phenotypic variation, however, the estimates are not significant. When all autosomes are fitted simultaneously (Figure 4) SSC2, SSC3

Table 3 Estimates of autosomal heritability for androstenone

\begin{tabular}{|c|c|c|c|c|c|}
\hline Chr & $\mathrm{h}_{\text {autosome }}^{2}$ & se & $p$-val & $\mathrm{h}_{\text {polygenic }}^{2}$ & se \\
\hline 1 & 0 & 0.04 & 1 & 0.38 & 0.06 \\
\hline 2 & 0.02 & 0.02 & 0.16 & 0.33 & 0.06 \\
\hline 3 & 0.02 & 0.02 & 0.16 & 0.34 & 0.06 \\
\hline 4 & 0 & 0.02 & 1 & 0.36 & 0.06 \\
\hline 5 & 0.06 & 0.03 & 0.00051 & 0.29 & 0.06 \\
\hline 6 & 0 & 0.03 & 1 & 0.37 & 0.06 \\
\hline 7 & 0 & 0.02 & 1 & 0.36 & 0.06 \\
\hline 8 & 0 & 0.02 & 1 & 0.36 & 0.06 \\
\hline 9 & 0 & 0.02 & 1 & 0.37 & 0.06 \\
\hline 10 & 0 & 0.02 & 1 & 0.36 & 0.06 \\
\hline 11 & 0 & 0.02 & 1 & 0.36 & 0.06 \\
\hline 12 & 0 & 0.02 & 0.38 & 0.35 & 0.06 \\
\hline 13 & 0.02 & 0.02 & 0.21 & 0.33 & 0.06 \\
\hline 14 & 0 & 0.02 & 1 & 0.36 & 0.06 \\
\hline 15 & 0 & 0.02 & 0.36 & 0.35 & 0.06 \\
\hline 16 & 0 & 0.02 & 1 & 0.36 & 0.06 \\
\hline 17 & 0 & 0.02 & 1 & 0.36 & 0.06 \\
\hline 18 & 0 & 0.01 & 1 & 0.36 & 0.06 \\
\hline
\end{tabular}

Testing strategy was to compare fitting a random polygenic effect (based on a GRM estimated using all genotyped SNPs across the genome) plus a random effect for variance attributed to SNPs from a single autosome with a reduced model fitting only the random polygenic effect. $P$-val is the corresponding $\mathrm{p}$ value based on the distribution of the LRT being between $X_{1}^{2}$ and a point mass of zero. $h^{2}$ autosome is an estimate of the heritability of the autosome, $h^{2}$ polygenic is an estimate of the heritability from the entire genome. and SSC13 explain 5\%, 3\% and 4\% of the genetic variation. The sum of autosomal estimates of genetic variation from LRTpoly is 0.12 (Table 3). The genetic variation explained by fitting all autosomes simultaneously was 0.29 (Additional file 2: Table S1), indicating that LRTpoly is conservative as might be expected as part of the individual autosomal heritabilities are absorbed by the overall genomic polygenic effect.

An alternative testing strategy is to fit all autosomes in a full model and then drop them one at a time for a reduced model (LRTdrop). A comparison of significance of autosomal heritability of androstenone using three testing strategies is given in Figure 5. Dropping a chromosome from the model including all the autosomes provides a test for whether genetic variance is associated with that particular chromosome whilst accounting for background polygenic effects on other chromosomes. This contrasts with the model containing only a single chromosome (LRTind in Figure 5) where the LRT and variance explained may be inflated by genetic variance from the rest of the genome that is not explicitly included in the model. For androstenone the results for LRTdrop suggest that chromosomes 2, 3, 5 and 13 explain a significant proportion of the variance.

\section{Discussion}

A genome-wide association study (GWAS) was carried out to identify SNPs associated with effects on androstenone and skatole in intact male pigs. The effect of the SIRI0000194 SNP on skatole estimated by fitting the genotypes as a covariate in the linear mixed model (3) was $5 \%$ of the phenotypic variance of the selected population (Table 2). The expectation in the general

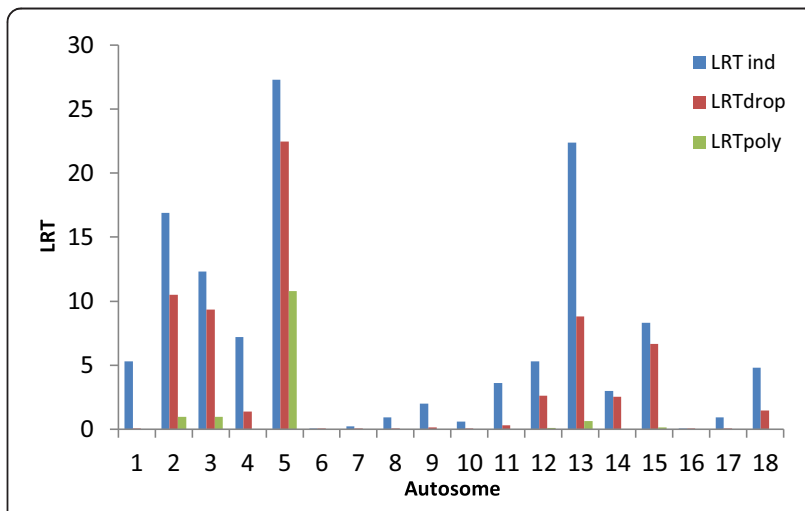

Figure 5 Likelihood ratio test (LRT) for significance of autosomal heritability or proportion of phenotypic variance explained for androstenone using three different linear mixed models. LRTind is comparing a model fitting an individual autosome with a null model, LRTdrop is where all autosomes are fitted and compared with a model which drops each autosome in turn, LRTpoly is comparing a model fitting an individual autosome plus a polygenic effect with a model containing only a polygenic effect. 
population assuming a heritability of 0.4 is that it would explain $\sim 12.5 \%$ of the genetic variation. The SIRI0000194 SNP, which was reported previously as AJ697882_2412 [32], is located within the promoter of the CYP2E1 gene. In a small separate sample of 83 Danish pigs significantly more AJ697882_2412 (SIRI0000194) CC homozygotes were observed in the 'high' skatole group [32]. More recently associations between skatole levels in two Duroc populations and the AJ697882_2412 (SIRI0000194) SNP have been reported [33]. Again the CC homozygotes exhibited the highest skatole levels. Although SIRI0000194 lies within a block of high LD (Figure 6) spanning several other genes there is evidence to support CYP2E1 as a candidate for the gene responsible for the observed associations with skatole levels. This gene has been previously identified as a candidate and is involved in the degradation of skatole in the liver where it is solely and abundantly expressed [36] (see also (http://biogps.org).

The GWAS for skatole was repeated, fitting the SNP SIRI000094 into the linear mixed model as a fixed effect. This model resulted in a change of ranking among the SNPs. The effect of greatest significance $(\mathrm{P}<0.001)$ was associated with SNP marker MARC0040638 located on chromosome 6 within the estradiol 17-beta-dehydrogenase 2 (HSD17B2) gene. The HSD17B2 gene and MARC0040638 SNP were located at SSC6:4,514,200-4,578,665 in an earlier genome assembly (Sscrofa9) but are located on unplaced scaffolds on the present assembly (Sscrofa10.2). The assignment of MARC0040638 SNP to SSC6 is confirmed from radiation hybrid mapping data (Additional file 2: Table S1 in [37]). Both the MARC0040638 SNP and $H S D 17 B 2$ gene are present in the sequence of the $\mathrm{CH} 242-$ 77H3 BAC clone (Genbank accession: CU929847). Incomplete sequence data from this BAC clone contribute to the current pig genome assembly (Sscrofa10.2) on SSC6 6.8766.939 Mbp. This SNP did not exceed the FDR or genomewide threshold, however a region on chromosome 6 spanning this gene was previously found to be significant for skatole in Landrace pigs [18]. Ramos et al., [18] found significant associations between skatole levels in Duroc pigs and SNPs mapping to a $6 \mathrm{Mbp}$ region on SSC6 corresponding to $1.829-8.498 \mathrm{Mbp}$ in Sscrofa10.2 coordinates and thus including the MARC0040638 SNP and HSD17B2 gene. In an earlier study, we mapped QTL for skatole, as detected by a (human) sensory panel, by linkage analysis with a low density microsatellite marker map with the closest marker SW1353 mapping to SSC6: 9.872 Mbp (Sscrofa10.2 coordinates) [13]. Human estradiol 17-betadehydrogenase 2 (HSD17B2) is involved in the synthesis of the 17 beta-hydroxysteroids: delta 5 -androstene- 3 beta, 17 beta-diol, testosterone, 17 beta-estradiol and dihydrotestosterone [38]. The HSD17B2 gene is thus important for steroid hormone synthesis and is abundantly expressed in pig liver, ureter and stomach (fundus), [36] (see also (http://biogps.org). Another 17-beta hydroxysteroid dehyrdorgenase gene (HSD17B7) has been examined as a candidate gene for an androstenone QTL on SSC4 [39].

A significant effect on androstenone was found associated with the H3GA0016037 SNP on chromosome 5 explaining $4 \%$ of the phenotypic variance. H3GA0016037 lies between the gene encoding transcription factor $N E U$ ROD4 neurogenic differentiation 4 and the TESPA1 thymocyte expressed positive selection association 1 locus. In humans TESPA1 is involved in the selection of thymocytes and T-cell development. It has been hypothesised that the production of glucocorticoid steroids may in some way regulate thymocyte selection [40]. The second most significant GWAS result was for ASGA0025097 which is located $\sim 4$ Mbp distal to the H3GA0016037 SNP on chromosome 5 . The genes of interest located within this $4 \mathrm{Mbp}$ region include the retinol dehydrogenase 5 (RDH5) and retinol dehydrogenase 16 (RDH16) genes. The $R D H$ gene encodes an enzyme which recognizes $5 \alpha-$ androstan- $3 \alpha, 17 \beta$-diol and androsterone as substrates and

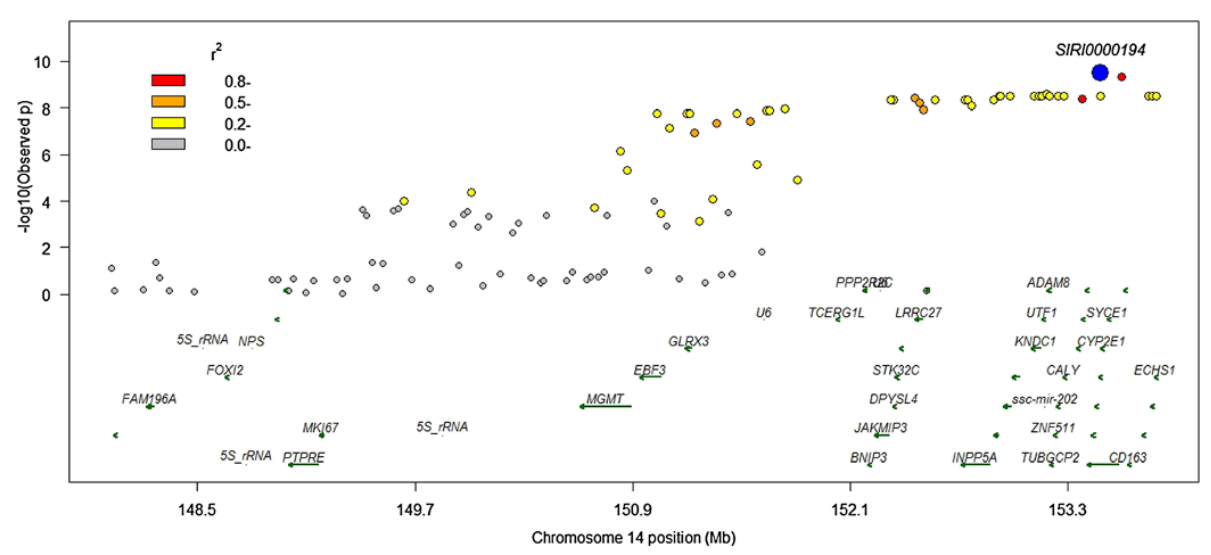

Figure 6 LD decay from SNP SIRI0000194 plotted against significance of effect on Skatole, pairwise LD in the region and genes located within the region. Sscrofa genome build 10.2 . 
is expressed in liver, testes and other tissues in humans [41]. $\mathrm{RDH16}$ is abundantly expressed in pig liver, testes and placenta [36] (see also http://biogps.org). Another 17beta hydroxysteroid dehyrdrogenase gene (HSD17B6) is located about 0.5 Mbp upstream of the ASGA0025097 SNP. The $4 \mathrm{Mbp}$ region between the two top SNPs is gene-rich and exhibits high levels of LD in the Danish Landrace population studied (Figure 3). Ironically, many of the genes in this region encode olfactory receptors. The minor allele frequency for both SNPs (ASGA0025097, H3GA0016037) was 0.14 and the $r^{2}$ between them was 0.68 . Fitting either SNP results in the loss of the effect indicating that both SNPs are tagging the same causal variant. This region has been found to be significant for androstenone measured in the fat of Duroc pigs, and for estradiol in Landrace pigs [16], however this region has not previously been found to be significant for effects on androstenone levels in Landrace.

Results from the regional heritability study reflected the GWAS analysis with the greatest heritability for androstenone on chromosome 5. This indicates that the regional approach successfully identifies autosomes with genetic variation attributable to the trait and that genetic variance is not correlated to the length of autosomes as seen by Yang et al. [24]. Here, the correlation of variance explained, with length of autosome, was $0.02(\mathrm{P}<0.93)$ for androstenone. There was evidence of information beyond the GWAS results from the regional approach. The method did point to an association of SSC2 and SSC3 with androstenone not seen in the GWAS. Highly significant effects for multiple QTL on these chromosomes associated with androstenone have been previously reported [11-13,16]. We cannot ascertain whether the SNP effect on SSC17 associated with androstenone is undetected by the regional approach or a spurious artifact of the GWAS. One approach might be using sequence information for imputation to increase the number of SNP genotypes and subsequently to divide the genome into many smaller regions providing greater resolution. Combined results of multiple SNP genotypes are less likely to yield spurious results from anomalies such as population stratification and differing minor allele frequencies at individual SNPs. The autosomes explaining the most variation have a greater likelihood for housing putative candidate genes and pathways. A further use for the estimated SNP or region effects in this population could be genomic prediction in unphenotyped individuals. This potential application is of particular relevance in traits that can only be measured post slaughter such as boar taint where phenotypes are of high economic impact and could result in rejection of the entire carcass.

\section{Conclusions}

Significant associations were found for skatole on SSC14 and for androstenone on SSC5 in Landrace pigs. The study agrees with a body of evidence that the CYP2E1 gene has effects on skatole breakdown in the liver. Autosomal heritability estimates agree with the GWAS and provide an opportunity to identify regions for further study. Differences between the GWAS and the autosomal heritability suggest that for androstenone there is variation explained by SSC2 and SSC3 that is not detected by the GWAS and that the SNP on chromosome 17 does not appear to contribute variance at the level of the autosome.

\section{Methods}

Animals

All the animals involved in this study were raised under conventional pig production conditions and were not subjected to any experimental procedures. All the samples for the study were collected post-mortem in a commercial abattoir.

\section{Taint measures}

Tissue fat samples were assayed for skatole levels using a calorimetric method in-house at the abattoir [42]. A second tissue sample taken about an hour after slaughter was subsequently assayed for androstenone by the Norwegian School of Veterinary Science using a modified time-resolved fluoroimmunoassay [43].

\section{Heritabilities}

A fixed effect of herd; and significant covariates meat percentage, slaughter weight and age at slaughter, were estimated using a linear mixed model in software package ASReml2 [44] (1). Fixed effects and covariates for skatole were estimated using the entire population of 6,000 animals in order to achieve the greatest possible accuracy. Heritabilities were estimated using pedigree relationships in the entire population of 6,000 individuals for Skatole and the 1,000 individuals phenotyped for androstenone.

$$
\mathbf{Y}=\mathbf{X} \boldsymbol{\beta}+\mathbf{Z u}+\mathbf{e}
$$

Where $\mathbf{Y}$ is an $n \times 1$, vector of $\log$ phenotype, $n$ is the number of individuals, $\mathbf{X}$ is an incidence matrix relating solutions for fixed effects of herd and covariates of age, mds co-ordinates contained in $\boldsymbol{\beta}$ to individuals, $\mathbf{u}$ is an $n \times 1$ vector of genetic effects, $\mathbf{Z}$ is an $n \times n$ incidence matrix relating individuals to genetic effects, and $\mathbf{e}$ is an $n \times 1$ vector of individual residual effects. $\mathbf{u} \sim \mathrm{N}\left(0, \mathbf{A} \sigma_{u}^{2}\right)$, and $\mathbf{e}$ is distributed as $\mathbf{e} \sim \mathrm{N}\left(0, \mathbf{I} \sigma_{\mathrm{e}}^{2}\right)$. $\mathbf{A}$ is the $n \times n$ genetic relationship matrix estimated from pedigree relationships.

\section{Genomic relationship matrices}

SNP genotypes were used to estimate shared coancestry or identity by state between individuals with rare SNPs weighted more heavily. The $n \times n$ genomic relationship 
matrix (GRM) of relatedness at a population level between $n$ individuals gives the covariance structure for the phenotype based on the premise that the more related two individuals are, or the greater the amount of the genome they share in common, the greater the expectation of phenotypic similarity. The proportion of alleles two individuals share in common are summed across all markers weighted by allele frequencies in the population in order to obtain an accurate estimate of how related two individuals are either across the entire genome or at a given region. Genomic relationship matrices were estimated using GenABEL [29] and GCTA [35] software.

Using the marker information for the 1,000 individuals, heritabilities were estimated by fitting the SNP based genomic relationship matrix from GenABEL in a linear mixed model to estimate polygenic effects from marker information (2). A genotypic correlation was estimated by a bivariate analysis of the two traits fitting the genomic relationship matrix using ASReml 2 software [44].

$$
\mathbf{Y}=\mathbf{X} \boldsymbol{\beta}+\mathbf{W g}+\mathbf{e}
$$

Where $\mathbf{g}$ is an $N \times 1$ vector of SNP effects, $N$ is the number of SNPs, W is an $n \times N$ incidence matrix relating SNP genotypes to $\mathbf{g}$. $\mathbf{G}$ is the $n \times n$ genomic relationship matrix estimated from SNP genotypes and $\mathbf{g} \sim \mathrm{N}\left(\mathbf{0}, \mathbf{G} \sigma_{g}^{2}\right)$.

\section{Association analysis}

Single SNP association tests were performed using a GRAM MAR [29] analysis (3) in GenABEL software. GRAMMAR uses a score test to identify associations between SNP genotypes and trait residuals after fixed and background genetic or polygenic effects are accounted for in the linear mixed model (2). Polygenic effects were estimated using a grm estimated from the average relationship between individuals at all SNP markers (weighted by allele frequency) across the genome.

$$
\begin{aligned}
& \mathbf{y}=\mathbf{S N P}+\mathbf{e} \\
& \lambda=\operatorname{Median}\left(\mathrm{T}_{1}^{2}, \mathrm{~T}_{2}^{2}, \ldots \ldots, \mathrm{T}_{\mathrm{N}}^{2}\right) / 0.456
\end{aligned}
$$

Where $\mathrm{T}$ is test statistic for N SNPs from (3).

Where $\mathbf{y}$ is a vector of trait residuals from (2), SNP is a vector of SNP genotypes and $\mathbf{e}$ is a vector of random residuals.

A correction factor or lambda $[29,45]$ was estimated from the distribution of test statistics to further account for systematic bias (4). A factor greater than 1 is indicative of systematic inflation of test results when compared to a distribution of the expectation under the null hypothesis. A factor less than one often results from over correction in a grammar analysis. The grammar function in GenABEL adjusts for this deflation factor. Permutation analysis $(100,000)$ was used to determine a rigorous threshold for genome-wide significance accounting for multiple testing and for any unaccounted for systematic bias. A less rigorous FDR cut off of $<0.05$ was applied to report SNPs of interest to aid the comparison of results from past and future study populations.

As grammar analyses tend to underestimate true SNP effects [29], genome-wide significant SNPs identified with the grammar analysis were fitted individually as covariates in the linear mixed model using ASReml 2 software to estimate SNP effects and to verify significance (5). The additive genetic variance was estimated as $2 p(1-p) \alpha^{2}$ where $p$ is the allele frequency for the most common SNP allele and $\alpha$ is the estimated effect. A further check was that this estimate was consistent with the difference in phenotypic variance when fitting, and not fitting, SNP genotype as a covariate in the LMM.

$$
\begin{aligned}
\mathrm{y}= & \mu+\text { herd }+\mathrm{b} 1 * \mathrm{SNP}+\mathrm{b} 2 * \text { slaughter weight } \\
& +\mathrm{b} 3 * \text { age }+\mathrm{b} 4 * \text { meat percentage } \\
& +\mathrm{mds}+a+\mathrm{e}
\end{aligned}
$$

Where $y=\log$ trait. Herd is fitted as a fixed effect. SNP genotype, slaughter weight, age, meat percentage and coordinates from the multi-dimensional scaling (mds) are fitted as covariates, $a$ is a random polygenic effect estimated using a SNP-based relationship matrix and e is the random residual.

\section{Estimation of regional genetic contribution or 'autosomal heritability'}

The linear mixed model (2) can be extended to divide phenotypic variance into estimates of the genetic and environmental variance containing information from genotypes of a group of $N$ SNPs spanning a region. This method has been implemented in the GCTA software package and it has been shown that the method can be used to estimate genetic variation for any region of the genome [35]. We divided the pig genome into the $18 \mathrm{au}-$ tosomes and estimated the contribution to heritability from each autosome (6). For these analyses only SNPs that mapped to Sscrofa 10.2 were used, any SNPs without a position on the current assembly were omitted as they could not be assigned to an autosome. Omitting these SNPs ( $\sim 13 \%$ of all SNPs) from the GRM made very little difference to the estimate of total genetic variance. The heritability estimate dropped by 0.0065 . This indicates that this subset of annotated SNPs was sufficiently large enough to accurately estimate relationships between individuals and to capture the genetic variance.

$$
\mathbf{Y}=\mathbf{X} \boldsymbol{\beta}+\sum_{c h r=1}^{18} \mathbf{W} \mathbf{u}_{c h r}+\mathbf{e}
$$


To avoid confounding of genetic variation of the trait and potential variation due to population stratification, eigenvectors were estimated from the genetic relationship matrix and the first 4 principal components fitted as covariates in the linear mixed model. This is slightly conservative and based on the results of the model based clustering described earlier which showed that the data forms 3 distinct clusters. The fixed effects and covariates of herd, age, meat percentage and slaughter weight were fitted into a linear mixed model together with eighteen variance components - one for each of the eighteen autosomes requiring 18 separate genetic relationship matrices to model the covariance structure and to partition the genetic variance into estimates of autosomal heritability.

To test the significance of individual autosomes a likelihood ratio test (LRT) comparing a model fitting the individual autosome plus a variance component for all SNPs in the grm (i.e. the equivalent of a genomic polygenic effect) was compared to a model fitting only the polygenic effect (LRTpoly). All SNPs were used in the polygenic effect to ensure that the models were truly nested. This conservative approach ensures that the variance explained by an autosome is not inflated by background polygenic effects.

Two further approaches were used. Firstly, comparing a model fitting a variance component estimated from the SNPs on a single autosome with a null model (LRTind). Secondly, a model fitting all 18 variance components compared with a model dropping each of the autosomes in turn (LRTdrop).

GCTA solves the linear mixed model (LMM) and obtains estimates of genetic and residual variances by restricted maximum likelihood (REML) using the average information (AI) algorithm.

A test statistic was obtained using a standard LRT statistic calculated as twice the difference between the log likelihoods of the full model and the null or reduced model that did not fit a genetic component. The LRT was tested against a chi square distribution. The LRT for one extra variance component is distributed as a mixture of point 0 and 1degrees of freedom (df) [46]. To account for this a $P$-value for a test assuming $1 \mathrm{df}$ was divided in two.

\section{Additional files}

Additional file 1: Figure S1. Distribution of skatole and androstenone measures and log transformed measures of skatole and androstenone.

Additional file 2: Table S1. Effect of testing structure on estimates and significance of autosomal heritability.

Additional file 3: Autosomal heritability analysis for skatole.

\section{Competing interests}

The authors declare they have no competing interests.

\section{Authors' contributions}

ALA, CSH and IV designed the study. IV coordinated sample collection. NHC managed and undertook laboratory work. CSH and DJK coordinated data analyses which were carried out by SJR, BL and BK. SJR prepared the manuscript. All authors contributed to the manuscript and approved the final manuscript.

\section{Acknowledgements}

These results are part of the SABRE research project that has been co-financed by the European Commission, within the 6th Framework Programme, contract No. FOOD-CT-2006-016250. The authors also acknowledge funding and support from the Meat and Livestock Commission. We are grateful to Jen Anderson and Heather Finlayson for technical support and to Brian McTeir for logistical support. We are grateful to the anonymous reviewers for their suggestions for improving this manuscript.

\section{Author details}

${ }^{1}$ The Roslin Institute and R(D)SVS, University of Edinburgh, Easter Bush, Midlothian, Scotland EH25 9RG, UK. 'Faculty of Agriculture, Department of Animal Science, Section of Biometry and Genetics, University of Akdeniz, Antalya 07059, Turkey. ${ }^{3}$ The Department of Animal Breeding and Genetics, Swedish University of Agricultural Sciences, Uppsala SE-750 07, Sweden. ${ }^{4}$ Faculty of Agriculture, University of J.J.Strossmayer in Osijek, Kralja Petra Svačića 1d, Osijek 31000, Croatia. ${ }^{5}$ Pig Research Centre, Danish Agriculture \& Food Council, Axeltorv 3, København V 1609, Denmark. ${ }^{6}$ MRC Human Genetics Unit, MRC IGMM, University of Edinburgh, Crewe Road, Edinburgh, Scotland EH4 2XU, UK. ${ }^{7}$ AgResearch, Dept of Animal Genomics, Invermay Agricultural Centre, Private Bag 50034, Puddle Alley, Mosgiel 9053, New Zealand.

Received: 7 March 2013 Accepted: 9 May 2014

Published: 3 June 2014

\section{References}

1. Patterson RLS: 5a-androst-16-en-3-one: compound responsible for taint in boar fat. J Sci Food Agric 1968, 19:31-38.

2. Desmoulin B, Bonneau M, Frouin A, Bidard JP: Consumer testing of pork and processed meat from boars: the influence of fat androstenone level. Livest Prod Sci 1982, 9:707-715.

3. Walstra P, Claudi-Magnussen C, Chevillon P, Von Seth G, Diestre A, Matthews KR, Homer DB, Bonneau M: An international study on the importance of androstenone and skatole for boar taint: levels of androstenone and skatole by country and season. Livest Prod Sci 1999, 62:15-28.

4. Malmfors $B$, Lundstrom $K$ : Consumer reactions to boar taint - a review. Livest Prod Sci 1983, 10:187-196.

5. Bonneau M, LeDenmat M, Vaudelet JC, Nunes JRV, Mortensen AB, Mortensen HP: Contributions of fat androstenone and skatole to boar taint: I. Sensory attributes of fat and pork meat. Livest Prod Sci 1992, 32:63-80.

6. Bonneau M, Kempster AJ, Claus R, Claudi-Magnussen C, Diestre A, Tornberg E, Walstra P, Chevillon P, Weiler U, Cook GL: An international study on the importance of androstenone and skatole for boar taint: I. Presentation of the programme and measurement of boar taint compounds with different analytical procedures. Meat Sci 2000, 54:251-259.

7. Melrose DR, Reed HCB, Patterson RL: Androgen steroids associated with boar odour as an aid to the detection of oestrus in pig artificial insemination. Br Vet J 1971, 127:497-502.

8. Keller A, Zhuang H, Chi Q, Vosshall LB, Matsunami H: Genetic variation in a human odorant receptor alters odour perception. Nature 2007, 449:468-472.

9. Lunde K, Egelandsdal B, Skuterud E, Mainland JD, Lea T, Hersleth M, Matsunami H: Genetic variation of an odorant receptor OR7D4 and sensory perception of cooked meat containing androstenone. PLOS One 2012, 7:e35259.

10. European Food Safety Authority: Opinion of the scientific panel on animal health and welfare on a request from the commission related to welfare aspects of the castration of piglets. EFSA Journal 2004, 91:1-18. http:// www.efsa.europa.eu/en/efsajournal/doc/91.pdf.

11. Robic A, Larzul C, Bonneau M: Genetic and metabolic aspects of androstenone and skatole deposition in pig adipose tissue: a review. Genet Sel Evol 2008, 40:129-143. 
12. Gregersen VR, Conley LN, Sørensen KK, Guldbrandtsen B, Velander $I H$, Bendixen C: Genome-wide association scan and phased haplotype construction for quantitative trait loci affecting boar taint in three pig breeds. BMC Genomics 2012, 13:22.

13. Lee GJ, Archibald AL, Law AS, Lloyd S, Wood J, Haley CS: Detection of quantitative trait loci for androstenone, skatole and boar taint in a cross between large white and meishan pigs. Anim Genet 2005, 36:14-22.

14. Varona L, Vidal O, Quintanilla R, Gil M, Sánchez A, Folch JM, Hortos M, Ruis MA, Amills M, Noguera JL: Bayesian analysis of quantitative trait loci for boar taint in a landrace outbred population. J Anim Sci 2005, 83:301-307.

15. Robic A, Le Mignon G, Fève K, Larzul C, Riquet J: New investigations around CYP11A1 and its possible involvement in an androstenone QTL characterised in Large White pigs. Genet Sel Evol 2011, 43:15.

16. Grindflek $E_{\text {, Lien }} \mathrm{S}$, Hamland $\mathrm{H}$, Hansen MH, Kent M, Van Son M, Meuwissen $\mathrm{TH}$ : Large scale genome-wide association and LDLA mapping study identifies QTLs for boar taint and related sex steroids. BMC Genomics 2011, 12:362.

17. Duijvesteijn N, Knol EF, Merks JW, Crooijmans RP, Groenen MA, Bovenhuis $H$, Harlizius B: A genome-wide association study on androstenone levels in pigs reveals a cluster of candidate genes on chromosome 6. BMC Genet 2010, 11:42

18. Ramos AM, Duijvesteijn N, Knol EF, Merks JW, Bovenhuis H, Crooijmans RP, Groenen MA, Harlizius B: The distal end of porcine chromosome $6 p$ is involved in the regulation of skatole levels in boars. BMC Genet 2011, 12:35.

19. Xue J, Dial GD, Holton EE, Vickers Z, Squires EJ, Lou Y, Godbout D, Morel N Breed differences in boar taint: relationship between tissue levels boar taint compounds and sensory analysis of taint. J Anim Sci 1996, 74:2170-2177

20. Doran E, Whittington FW, Wood JD, McGivan JD: Cytochrome P450IIE1 (CYP2E1) is induced by skatole and this induction is blocked by androstenone in isolated pig hepatocytes. Chem Biol Interact 2002, 140:81-92.

21. Lander ES: Initial impact of the sequencing of the human genome. Nature 2011, 470:187-197.

22. Manolio TA, Collins FS, Cox NJ, Goldstein DB, Hindorff LA, Hunter DJ, McCarthy MI, Ramos EM, Cardon LR, Chakravarti A, Cho JH, Guttmacher AE, Kong A, Kruglyak L, Mardis E, Rotimi CN, Slatkin M, Valle D, Whittemore AS, Boehnke M, Clark AG, Eichler EE, Gibson G, Haines JL, Mackay TF, McCarroll SA, Visscher PM: Finding the missing heritability of complex diseases. Nature 2009, 461:747-753.

23. Nagamine $Y$, Pong-Wong R, Navarro P, Vitart V, Hayward C, Rudan I, Campbell H, Wilson J, Wild S, Hicks AA, Pramstaller PP, Hastie N, Wright AF, Haley CS: Localising loci underlying complex trait variation using regional genomic relationship mapping. PLoS One 2012, 7:e46501.

24. Yang J, Manolio TA, Pasquale LR, Boerwinkle E, Caporaso N, De Cunningham JM, Andrade M, Feenstra B, Feingold E, Hayes MG, Hill WG, Landi MT, Alonso A, Lettre G, Lin P, Ling H, Lowe W, Mathias RA, Melbye M, Pugh E, Cornelis MC, Weir BS, Goddard ME, Visscher PM: Genome partitioning of genetic variation for complex traits using common SNPs. Nat Genet 2011, 43:519-525.

25. Chen Z, Zheng G, Ghosh K, Li Z: Linkage disequilibrium mapping of quantitative-trait Loci by selective genotyping. Am J Hum Genet 2005, 77:661-669.

26. Darvasi A, Soller M: Selective genotyping for determination of linkage between a marker locus and a quantitative trait locus. Theor Appl Genet 1992, 85:353-359.

27. Tajet $\mathrm{H}$, Andresen $\mathrm{O}$, Meuwissen $\mathrm{T}$ : Estimation of genetic parameters of boar taint; skatole and androstenone and their correlations with sexual maturation. Acta Vet Scand 2006, 48(Suppl 1):S9.

28. Ramos AM, Crooijmans RP, Affara NA, Amaral AJ, Archibald AL, Beever JE, Bendixen C, Churcher C, Clark R, Dehais P, Hansen MS, Hedegaard J, Hu ZL, Kerstens HH, Law AS, Megens HJ, Milan D, Nonneman DJ, Rohrer GA, Rothschild MF, Smith TP, Schnabel RD, Van Tassell CP, Taylor JF, Wiedmann RT, Schook LB, Groenen MA: Design of a high density SNP genotyping assay in the pig using SNPs identified and characterized by next generation sequencing technology. PLoS One 2009, 4:e6524.

29. Aulchenko YS, Ripke S, Isaacs A, Van Duijn CM: GenABEL: an R library for genome-wide association analysis. Bioinformatics 2007, 23:1294-1296.
30. R Core Team; R: A language and environment for statistical computing. In R Foundation for Statistical Computing. Vienna, Austria: R Core Team; R; 2013. http://www.R-project.org.

31. Groenen MA, Archibald AL, Uenishi H, Tuggle CK, Takeuchi Y, Rothschild MF Rogel-Gaillard C, Park C, Milan D, Megens HJ, Li S, Larkin DM, Kim H, Frantz LA, Caccamo M, Ahn H, Aken BL, Anselmo A, Anthon C, Auvil L, Badaoui B, Beattie CW, Bendixen C, Berman D, Blecha F, Blomberg J, Bolund L, Bosse M, Botti S, Bujie Z, et al: Analyses of pig genomes provide insight into porcine demography and evolution. Nature 2012, 491:393-398.

32. Skinner TM, Doran E, McGivan JD, Haley CS, Archibald AL: Cloning and mapping of the porcine cytochrome-p450 2E1 gene and its association with skatole levels in the domestic pig. Anim Genet 2005, 36:417-422.

33. Mörlein D, Lungershausen $M$, Steinke $K$, Sharifi AR, Knorr C: A single nucleotide polymorphism in the CYP2E1 gene promoter affects skatole content in backfat of boars of two commercial Duroc-sired crossbred populations. Meat Sci 2012, 92:739-744.

34. Squires EJ, Lundström K: Relationship between cytochrome P450IIE1 in liver and levels of skatole and its metabolites in intact male pigs. J Anim Sci 1997, 75:2506-2511.

35. Yang J, Lee SH, Goddard ME, Visscher PM: GCTA: a tool for genome-wide complex trait analysis. Am J Hum Genet 2011, 88:76-82.

36. Freeman TC, Ivens A, Baillie JK, Beraldi D, Barnett MW, Dorward D, Downing A, Fairbairn L, Kapetanovic R, Raza S, Tomoiu A, Alberio R, Wu C, Su Al, Summers KM, Tuggle CK, Archibald AL, Hume DA: A gene expression atlas of the domestic pig. BMC Biol 2012, 10:90.

37. Servin B, Faraut T, lannuccelli N, Zelenika D, Milan D: High-resolution autosomal radiation hybrid maps of the pig genome and their contribution to the genome sequence assembly. BMC Genomics 2012, 13:585.

38. Labrie Y, Durocher F, Lachance Y, Turgeon C, Simard J, Labrie C, Labrie F: The human type II 17 beta-hydroxysteroid dehydrogenase gene encodes two alternatively spliced mRNA species. DNA Cell Biol 1995, 14:849-861.

39. Chen G, Bourneuf E, Marklund S, Zamaratskaia G, Madej A, Lundström K: Gene expression of 3beta-hydroxysteroid dehydrogenase and 17betahydroxysteroid dehydrogenase in relation to androstenone, testosterone, and estrone sulphate in gonadally intact male and castrated pigs. $J$ Anim Sci 2007, 85:2457-2463.

40. Ashwell JD, Vacchio MS, Galon J: Do glucocorticoids participate in thymocyte development? Immunol Today 2000, 21:644-646.

41. Wang J, Chai X, Eriksson U, Napoli JL: Activity of human 11-cis-retinol dehydrogenase (Rdh5) with steroids and retinoids and expression of its mRNA in extra-ocular human tissue. Biochem J 1999, 338:23-27.

42. Hansen-Møller J, Andersen JR: Boar taint-analytical alternatives. Fleischwirtsch 1994, 74:963-966.

43. Tuomola M, Harpio R, Knuuttila P, Mikola H, Lövgren T: Time-resolved fluoroimmunoassay for the measurement of androstenone in porcine serum and fat samples. J Agric Food Chem 1997, 45:3529-3534.

44. Gilmour AR, Cullis BR, Harding SA, Thompson R: ASRem/ Update: What's new in Release 2.00. Hemel Hempstead, UK: VSN International Ltd; 2006.

45. Ekine CC, Rowe SJ, Bishop SC, De Koning DJ: What is the best phenotype for genome-wide association studies in data with defined pedigrees? In Proceedings of the 9th World Congress of Genetics Applied to Livestock Production. 2010:pp1-pp6.

46. Self SG, Liang KY: Asymptotic properties of maximum likelihood estimators and likelihood ratio tests under nonstandard conditions. J Am Stat Assoc 1987, 82:605-610.

\section{doi:10.1186/1471-2164-15-424}

Cite this article as: Rowe et al:: Analysis of the genetics of boar taint reveals both single SNPs and regional effects. BMC Genomics 2014 15:424. 